rev.relac.int.estrateg.segur.5(2):139-159,2010

\title{
LOS DESAFÍOS DEL ENVEJECIMIENTO POBLACIONAL PARA LAS POLÍTICAS PÚBLICAS EN MÉXICO*
}

\author{
Jorge Enrique Horbath** \\ Amalia Gracia**
}

\section{RESUMEN}

Al considerar las transformaciones demográficas experimentadas por la población mexicana en las últimas décadas, la deuda social acumulada se vuelve aún más apremiante, pues se está agotando el tiempo de «rentabilidad» que hubiera podido coadyuvar a proporcionar el «Bono Demográfico». El envejecimiento de la población evidencia la necesidad de

* El documento es parte del proyecto «Transferencias Intergeneracionales Asociadas al Cambio de la Estructura por Edad de la Población» Ilevado adelante por el Consejo Nacional de Población (CONAPO), en el marco del proyecto «Transferencias Intergeneracionales, Envejecimiento de la Población y Protección Social en América Latina» financiado por CEPAL-CELADE. Agradecemos al Mtro. Juan Cruz Olmeda, por sus valiosos aportes en el desarrollo del proyecto.

** Profesor-Investigador Titular del Centro de Estudios Políticos y Gestión Pública de El Colegio de Sonora (COLSON); miembro del Sistema Nacional de Investigadores (S.N.I.) Nivel 1 del Consejo Nacional de Ciencia y Tecnología de México (CONACYT). jhorbath@colson.edu.mx

*** Profesora-Investigadora Titular del Centro de Estudios del DesarroIlo de El Colegio de Sonora (COLSON); miembro del Sistema Nacional de Investigadores (S.N.I.) Nivel 1 del Consejo Nacional de Ciencia y Tecnología de México (CONACYT).mgracia@colson.edu.mx 
reformas políticas que no solamente cubran el rezago en materia de servicios sociales sino que otorguen garantías a una población que deberá soportar mayores tasas de dependencia en un contexto donde la pobreza no ha disminuido significativamente, pese a los esfuerzos de los novedosos programas implementados. Como producto de la fragmentación de la Política Pública, actualmente se le otorga a la política social el reto de responder por las grietas que ha ido dejando la política económica. Sin embargo, si no se conciben políticas que integren esta escisión y no se revierten los rumbos seguidos hasta aquí, el tan mentado bono terminará siendo una pesada factura a pagar con mayor desigualdad y pobreza.

Palabras clave: Envejecimiento poblacional; Políticas Públicas; Transferencia Intergeneracional.

\begin{abstract}
Considering the demographic changes experienced by the Mexican population in recent decades, the accumulated social debt becomes even more urgent as time is running time of «return» that would have been able to contribute to providing the «demographic bonus». The aging of the population highlights the need for political reforms that not only cover the backlog in social services but give guarantees to a population that will support higher rates of dependency in a context where poverty has not declined significantly, despite efforts of innovative programs implemented. As a result of the fragmentation of Public Policy, currently being given to social policy the challenge posed by the cracks which has left economic policy. However, if no policies are designed to integrate this division and are not followed the directions are reversed here, the much-vaunted voucher will end up being a heavy bill to pay greater inequality and poverty.
\end{abstract}

Words keys: Population aging; Public Policy, Intergenerational Transfer.

\title{
INTRODUCCIÓN
}

Durante las últimas décadas la población mexicana ha experimentado cambios sustantivos en su composición por grupos de edad, principalmente como consecuencia de la reducción paralela de las tasas de natalidad y mortalidad. La combinación de dichas tendencias ha dado lugar al inicio de un paulatino pero sostenido proceso de envejecimiento poblacional. Si hasta la actualidad esta dinámica daba la oportunidad de un bono demográfico para consolidar el desarrollo productivo, en el mediano y largo plazo la continuación de esta tendencia implicará un incremento sostenido de la proporción de adultos mayores junto a la reducción de la base de la pirámide poblacional.

El objetivo del presente artículo es analizar los desafíos particulares que plantean los mencionados cambios a las políticas públicas, haciendo hincapié en las políticas sociales dirigidas a ciertos 
grupos etarios de la población. En primer lugar, se realizan referencias conceptuales sobre las políticas públicas, los diferentes tipos y su papel para garantizar los derechos de las y los ciudadanos. Seguidamente, se analizan las tendencias que explican el envejecimiento poblacional en el caso de México y se consideran sus posibles efectos a largo plazo en los ingresos individuales y de las familias. Finalmente, se presta particular atención a las nuevas demandas que dichas transformaciones plantean para los sistemas de seguridad social y de atención de la salud orientados al segmento de adultos mayores, así como las oportunidades que la reducción paulatina del segmento de niños y niñas tiene para el desarrollo de políticas educativas de mayor calidad.

\section{DEFINICIONES GENERALES Y PARÁMETROS PARA DIFERENCIAR LAS POLÍTICAS PÚBLICAS}

Aproximarnos a las políticas públicas supone dar cuenta de su razón de ser y ámbito de acción. La idea misma de políticas públicas habla de la existencia de una esfera de la vida que excede lo privado o puramente individual y remite a la existencia de una colectividad y en un espacio en la que esta pueda desarrollarse. (Parsons, 2007). Los límites y alcances de «lo público» se definen socialmente y, como tal, se han ido redefiniendo a lo largo del tiempo. Considerando lo anterior y de modo siempre relativo y transitorio, podemos establecer que «lo público comprende aqueIla dimensión de la actividad humana que se cree que requiere la regulación o intervención gubernamental o social, o por lo menos la adopción de medidas comunes» (Parsons, 2007).

Existe una diversidad de definiciones de política pública. En términos generales y clásicos, se observa que una política estatal supone una toma de posición que intenta alguna forma de resolución de una cuestión surgida del seno de la sociedad (Oszlak y O’Donnell, 1976). Por cuestión se entiende a los asuntos (demandas, necesidades) que están socialmente problematizados o que se han incorporado a la agenda de discusión pública. Las políticas públicas, entonces, refieren a las decisiones de una o más agencias estatales e implican un conjunto de acciones y omisiones que manifiestan una determinada modalidad de intervención del Estado en relación con una cuestión socialmente problematizada. (Ozslak y O’Donnell, 1976). El primer paso para la formulación de una política estatal es justamente el proceso por medio del cual cierta cuestión pasa de la agenda pública a la agenda de gobierno. Este pasaje requiere que una particular demanda o necesidad forme parte desde entonces de los problemas, cuestiones o asuntos que los tomadores de decisión han definido como los objetivos de su acción y, más precisamente, como demandas o necesidades a las que han decidido dar respuestas (Aguilar Villanueva, 1995). En esta línea se inscribe la definición de Thoenig, quien propone que las políticas públicas refieren a «lo que los gobiernos deciden o no realizar». A partir de estas consideraciones generales surge una serie de temáticas que es oportuno plantear aquí.

En primer lugar, debe destacarse que el hecho de que una demanda particular devenga prioritaria en la agenda de gobierno constituye un proceso complejo durante el cual una serie de actores - 
con diferente grado de influencia y acceso a recursos de poder- toma posición y ejerce su influencia sobre la arena política. Esto significa que en dicho proceso existen relaciones de fuerza particulares que no sólo determinan qué tipo de necesidad llega a la agenda sino también el sentido que ella adquiere y las repuestas que genera. Tal como observa Parsons (2007), la definición del problema que la política pública atenderá constituye parte del problema y supone una cierta interpretación de la realidad que moldea el tipo de política o «solución» para resolverlo.

Por otro lado, es necesario no olvidar que el Estado no constituye una entidad monolítica, por lo cual su toma de posición frente a una determinada cuestión puede no resultar ni homogénea ni unívoca ni permanente. Ciertas tomas de posición implican intensos procesos de negociación dentro del Estado al tiempo que pueden darse situaciones en las que diferentes agencias se superpongan sin coordinación en la generación de políticas orientadas a atender una misma cuestión. Asimismo, políticas que apunten a atender cuestiones particulares de una determinada área pueden -al no tomar en cuenta otras líneas de política impulsadas por otras agencias gubernamentales- generar externalidades que impacten negativamente en otras áreas de política, limitando las posibilidades de éxitos de dichas políticas o dando lugar -de manera indirectaal surgimiento de nuevas necesidades anteriormente no atendidas.

En conexión con lo anterior, Kauffer Michel (2002) señala que las autoridades estatales son los principales actores de la política pública, pero que dichas políticas no pueden ser entendidas ni explicadas con prescindencia de las estrategias llevadas adelante por otros actores como organizaciones, grupos e incluso, individuos estratégicamente ubicados en el sistema de poder. Pese a que la acción del Estado aparece como sustantivamente diferente en tanto está investida de un carácter obligatorio, esta se inserta en un entramado en el cual se interrelaciona con las acciones (e inacciones) de los otros actores partícipes del juego ${ }^{1}$.

Finalmente, al formularse con base en una problemática particular, las políticas públicas se diseñan por lo general con la intención de producir efectos en un determinado grupo poblacional, perjudicado por el tema en cuestión, cuyos efectos la política se propone revertir. La relación entre las políticas particulares y la población está atravesada por diferentes problemáticas y consideraciones, que iremos abordando en los próximos apartados.

\section{Algunas categorías para diferenciar los tipos de política.}

Una primera y posible aproximación a la diferenciación de las políticas públicas implementadas por el Estado puede ser identificarlas según el tipo de asunto o problema al que estas pretenden

\footnotetext{
1 Vale la pena aclarar que estas interacciones tienen lugar en cada una de las etapas del ciclo de las políticas tal como lo define Palumbo (1987). Entre dichas etapas podemos identificar: establecimiento de la agenda; definición del problema; diseño de la política; legitimación de la política; implementación; impacto; terminación (Palumbo, 1987).
} 
atender. Este acercamiento, esencialmente descriptivo, asume la posibilidad de distinguir analíticamente diversas esferas de la vida pública. Desde este enfoque es posible establecer subtipos de políticas de acuerdo a si estas apuntan a abordar problemáticas ligadas al área económica, el área de defensa, el área de seguridad y así sucesivamente.

Un acercamiento un tanto más sofisticado sería establecer una categorización de las políticas de acuerdo con su impacto real o esperado en la sociedad (Lowi, 1964). Este enfoque apunta a superar una visión meramente descriptiva reemplazándola por un análisis que destaca el aspecto funcional de las políticas. Siguiendo la clásica clasificación de Lowi $(1964,1972)$ es posible hablar de: (a) políticas distributivas, (b) políticas regulatorias, (c) políticas redistributivas y (d) políticas constitutivas.

Las políticas distributivas hacen referencia al establecimiento de una serie de privilegios por parte del Estado con base en conductas de individuos sin que necesariamente se establezcan obligaciones para estos $u$ otros grupos de individuos; las políticas de subsidios constituyen un ejemplo paradigmático de esta categoría. Las políticas regulatorias, por su parte, implican el establecimiento por parte del Estado de reglas generales que intentan influir en el comportamiento y las conductas de los individuos mediante el uso de sanciones que penan las conductas transgresoras, elevando entonces los costos que ciertos cursos de acción tendrían de no cumplir dichas reglas; las políticas de combate a la corrupción, por ejemplo, pueden ser incluidas dentro de esta categoría. En cuanto a las políticas redistributivas, se puede decir que suponen la transferencia de recursos, a partir de la acción coercitiva del Estado, de un grupo de individuos a otros; dentro de esta categoría se incluyen todas las políticas que otorgan beneficios o concesiones a ciertos individuos cuyos costos tendrán que ser financiados por otros. Finalmente, las políticas constitutivas hacen referencia a acciones del Estado que apuntan a establecer las reglas básicas a partir de las cuales se reparte el poder en un determinado marco societal, redefiniendo las condiciones del sistema político o administrativo; aquí se incluyen las políticas orientadas a la creación de instituciones o agencias gubernamentales, así como aquellas que apunten a generar nuevos marcos normativos.

Una forma complementaria para distinguir a las políticas supone identificar el grupo de población al que se propone interpelar. Dicho segmentado de población puede definirse por ciertas características estructurales (como el grupo etario, sexo, cultura, religión, entre otras) o por compartir una serie de carencias que la política en cuestión intente subsanar (pobreza, desempleo, carencias nutricionales, por ejemplo). Aún si algunas políticas afectan a la población en su conjunto porque toman al todo social como eje de su accionar, pueden tener tanto efectos indirectos o no buscados explícitamente sobre ciertos segmentos poblacionales (como en el caso de algunas políticas macroeconómicas que, al impactar en variables económicas generales como la inflación o el tipo de cambio, generan efectos particulares en determinados sectores de la población), así como efectos directos y deseados (como en el caso de las políticas de población). 


\section{LAS POLÍTICAS PÚBLICAS COMO HERRAMIENTAS PARA GARANTIZAR EL PLENO EJERCICIO DE LOS DERECHOS}

Una vez consideradas las definiciones básicas que permiten clarificar de qué se habla cuando se hace referencia a las políticas públicas, surge el interrogante sobre «para qué» se efectúan dichas acciones o sobre las prioridades que estas deben atender. En este apartado se propone una serie de reflexiones sobre lo que consideramos fundamental para las políticas públicas: la generación de condiciones que aseguren el pleno ejercicio de derechos de los individuos.

Habiendo transitado la primera década del siglo XXI nos encontramos ante el desafío de dar cuenta de una serie de problemáticas que afectan a la población latinoamericana en lo referido a la satisfacción de las necesidades básicas y a las condiciones indispensables para ejercer plenamente sus derechos. La necesidad de transformar el crecimiento económico en desarrollo se plantea como una meta que los estados latinoamericanos deben incluir como prioritaria en sus agendas.

Aun con matices, es posible afirmar que la consolidación de regímenes democráticos en la región ha llevado a un paulatino aseguramiento de las pautas que dan garantías para el ejercicio de los derechos civiles y políticos. Sin embargo, y en el marco de importantes transformaciones experimentadas desde finales de la década de los ochenta del siglo XX en la matriz económica de la región, la realidad latinoamericana muestra aún un profundo déficit en relación a los derechos económicos, sociales y culturales (DESC) de sus habitantes ${ }^{2}$. Entre la mencionada categoría de derechos, reconocidos por diferentes pactos internacionales a los que los estados latinoamericanos han adherido ${ }^{3}$, pueden identificarse el derecho a la educación, el derecho a la seguridad social, el derecho a la salud física, mental y social, el derecho a la protección de los ancianos, entre otros.

En las últimas décadas los países de la región han experimentado un aumento en las necesidades básicas insatisfechas de la población, situación que evidencia la vulneración de los derechos básicos antes mencionados. Entre las tendencias que evidencian esta realidad destacan la consolidación de escenarios de pobreza estructural y exclusión social; las transformaciones experimentadas por los mercados laborales; las dificultades fiscales experimentadas por los Estados latinoamericanos y el consecuente deterioro en los niveles de cobertura de la seguridad social y la calidad de los servicios públicos, así como la erección de barreras en el acceso a la educación y la salud a partir de la proliferación de mecanismos de mercado en la provisión de dichos servicios.

2 Esto a pesar del mejoramiento que durante los últimos años han experimentado los indicadores relacionados a pobreza e indigencia en la región. Según cifras de la Cepal (2007), aún 190 millones de latinoamericanos vivían en situación de pobreza en 2006.

3 Entre los instrumentos de derecho internacional fundamentales deben destacarse el Pacto Internacional de Derechos Económicos, Sociales y Culturales (Pidesc), aprobado por la ONU en 1966, y el Protocolo de San Salvador de 1998 que amplía el conjunto de DESCs para la región. 
En este marco, para evaluar el éxito o fracaso de las políticas es cada vez más importante colocar como parámetro principal el cumplimiento de los derechos. Desde «...un enfoque de derechos humanos, los planes, las políticas y los procesos de desarrollo están anclados en un sistema de derechos y de los correspondientes deberes establecidos por el derecho internacional» (Oficina del Alto Comisionado, 2006:15) ${ }^{4}$. Esta conceptualización se sustenta en la noción de que el Estado tiene la obligación de respetar, proteger y satisfacer el goce de los mencionados derechos. Respetar supone que el Estado no debe interferir ni limitar la libre acción de los individuos en el uso de sus recursos propios y de las colectividades, y proteger implica que debe garantizar que los derechos no sean violados o limitados por la acción de terceros individuos. Mientras que para el efectivo cumplimiento de estas dos funciones el Estado está principalmente obligado a generar un marco jurídico que asegure el respeto a la individualidad, la obligación de satisfacer determina la adopción de una actitud proactiva. Al asumir dicha responsabilidad, el Estado reconoce su obligación de «...asegurar de manera plena el disfrute de los derechos...» y para lograrlo debe «... adoptar las medidas necesarias para garantizar a todas las personas que se encuentran bajo su jurisdicción la oportunidad de satisfacer adecuadamente las necesidades reconocidas en los instrumentos de derechos humanos, que no pueden alcanzar mediante el esfuerzo personal» (Oficina del Alto Comisionado 2003:61).

Teniendo en cuenta lo antedicho, la implementación de diferentes líneas de políticas públicas en especial las políticas económicas y las sociales- se convierte en el instrumento fundamental a partir del cual el Estado puede llevar a cabo la función de garante de los derechos. La Declaración de Quito explícitamente propone que «...los instrumentos internacionales de derechos económicos, sociales y culturales no imponen fórmulas uniformes, pero requieren al menos que el Estado arbitre los medios a su alcance para cubrir las necesidades mínimas de la población en las áreas involucradas y defina políticas de mejoramiento progresivo del nivel de vida de los habitantes, mediante la ampliación del disfrute de esos derechos» (Oficina del Alto Comisionado 2003:61-62).

En el caso de México, además de constar en los tratados internacionales signados a los que el país ha adherido ${ }^{5}$, la mayoría de dichos derechos se encuentran consagrados constitucionalmente y reafirmados por normativas específicas. Así, la Constitución destaca el derecho a la educación (Art. 3으), el derecho a la protección de la salud (Art.4ㅇ) , el derecho a un medio

4 Vale la pena aclarar que la adopción de un enfoque centrado en los derechos humanos no sólo se sostiene desde argumentos de carácter normativo sino que es posible sugerir que dicho acercamiento es asimismo preferible desde un punto de vista más pragmático o instrumental, pues políticas sustentadas en dicho enfoque producen resultados mejores y más sostenibles en materia de desarrollo humano.

5 Además de las ya mencionadas cabe desatacar la «Declaración del Milenio», aprobada por los jefes de Estado en el marco de la Asamblea General de la Organización de las Naciones Unidas que tuvo lugar en agosto de 2000. En dicha Declaración se establece el compromiso conjunto de los suscribientes para alcanzar una serie de metas en relación al desarrollo humano para el año 2015 
ambiente adecuado para el desarrollo y bienestar (Art. 4 ㅇ), el derecho de los niños y niñas a satisfacer sus necesidades de alimentación, salud, educación y sano esparcimiento (Art. 4으), el derecho a disfrutar de una vivienda digna y decorosa (Art 4으), el derecho al trabajo digno y útil socialmente (Art. 123을 y el derecho a salarios mínimos generales suficientes (Art. 123을.

En consonancia con la Carta Magna, la Ley General de Desarrollo Social (2004) establece en su Artículo 1ํ que dicha normativa se dicta con el objeto de "Garantizar el pleno ejercicio de los derechos sociales consagrados en la Constitución Política de los Estados Unidos Mexicanos, asegurando el acceso de toda la población al desarrollo social» (Inc. I), siendo que la Política Nacional de Desarrollo Social debe tener como objetivo «Propiciar las condiciones que aseguren el disfrute de los derechos sociales, individuales o colectivos, garantizando el acceso a los programas de desarrollo social y la igualdad de oportunidades, así como la superación de la discriminación y la exclusión social» (Art. 11으, Inc. I). En tanto el Artículo 6을 establece que «Son derechos para el desarrollo social la educación, la salud, la alimentación, la vivienda, el disfrute de un medio ambiente sano, el trabajo y la seguridad social y los relativos a la no discriminación en los términos de la Constitución Política de los Estados Unidos Mexicanos».

Asimismo, la Ley para la Protección de los Derechos de Niños, Niñas y Adolescentes (2000) establece como su principal objetivo «garantizar a niñas, niños y adolescentes la tutela y el respeto de los derechos fundamentales reconocidos en la Constitución» (Art. 1으), siendo que la protección de dichos derechos supone asegurar a niños, niñas y adolescentes «un desarrollo pleno e integral, lo que implica la oportunidad de formarse física, mental, emocional, social y moralmente en condiciones de igualdad». (Art. 3으). El Artículo 4을 en tanto especifica que «El ejercicio de los derechos de los adultos no podrá, en ningún momento, ni en ninguna circunstancia, condicionar el ejercicio de los derechos de niñas, niños y adolescentes».

Por su parte, la más reciente Ley de los Derechos de las Personas Adultas Mayores (2008) se propone como el medio para «garantizar el ejercicio de los derechos de las personas adultas mayores, así como establecer las bases y disposiciones para su cumplimiento» (Art. 10). En tanto, dichos derechos abarcan, entre otros, el derecho a una vida con calidad (Art. 5으, Inc. I a), a la protección contra toda forma de explotación (Art. 5ํ, Inc. I e), a tener acceso a los satisfactores necesarios «considerando alimentos, bienes, servicios y condiciones humanas o materiales para su atención integral» (Art. 5ㅜㅡ, Inc. III a), a tener acceso preferente a los servicios de salud (Art. 5ㅜㅡ, Inc. III b) y educación (Art. 50 Inc. IV a). El Artículo $6^{\circ}$ asimismo dispone que «El Estado garantizará las condiciones óptimas de salud, educación, nutrición, vivienda, desarrollo integral y seguridad social a las personas adultas mayores. Asimismo, deberá establecer programas para asegurar a todos los trabajadores una preparación adecuada para su retiro.»

Por último, La Ley General de Educación (1993) establece que «Todo individuo tiene derecho a recibir educación y, por lo tanto, todos los habitantes del país tienen las mismas oportunidades de acceso al sistema educativo nacional, con sólo satisfacer los requisitos que establezcan las dispo- 
siciones generales aplicables» (Art. $2^{\circ}$ ), siendo que «El Estado está obligado a prestar servicios educativos para que toda la población pueda cursar la educación preescolar, la primaria y la secundaria» (Art. 3을. Los artículos $5 \underline{0}$ y $6^{0}$ garantizar a su vez el carácter laico y gratuito de dicha educación. La Ley General de Salud (1984) establece lo propio en relación al ejercicio del derecho a la salud, destacando que dicho derecho supone, entre otras cuestiones, «La protección y el acrecentamiento de los valores que coadyuven a la creación, conservación y disfrute de condiciones de salud que contribuyan al desarrollo social» (Art. 2º, Inc. III).

Este marco normativo pone en evidencia el ineludible rol que el Estado mexicano debe asumir como garante de los derechos y establece el horizonte que debería guiar la acción de las políticas públicas. El proponer un acercamiento que privilegie este enfoque requiere clarificar una serie de elementos que permitan definir programas y políticas concretas. A esto nos dedicaremos en los próximos apartados.

\section{ENVEJECIMIENTO POBLACIONAL: ELEMENTOS PARA ENTENDER EL PRESENTE Y VISLUMBRAR EL FUTURO}

Según el Consejo Nacional de Población (Conapo, 2008), el descenso sostenido de la mortalidad es un fenómeno observable desde la finalización misma de la Revolución Mexicana. Entre 1930 y 1943 la tasa bruta de mortalidad disminuyó de 26.9 a 21.4, mientras que para 1960 dicho indicador se había reducido a la mitad (ubicándose en 12.8) para continuar su descenso hasta principios de siglo XXI (hasta representar 5). Paralelamente, la tasa de fecundidad continuó creciendo hasta mediados de los setenta, período a partir del cual, y como consecuencia de la difusión del uso de métodos anticonceptivos, se verifica un brusco y sostenido descenso. Entre principios de la década de 1960 y el inicio del siglo XXI la tasa de fecundidad -nacidos vivos por cada mil habitantes- se redujo desde más de 45 para 1960 a 25 en 2000. (Conapo, 2008).

En términos generales, la combinación de ambas tendencias implicó durante la segunda parte del siglo XX un sostenido crecimiento poblacional y un aumento significativo de la esperanza de vida. La tasa de crecimiento anual se elevó hasta un 3\% hacia finales de los cuarenta y continuó ubicándose incluso por arriba de dichos valores hasta finales de los años setenta. Dicha tasa muestra sin embargo valores más reducidos en años recientes: en 2000 el crecimiento anual se daba a un ritmo del 1.96\% mientras que para 2008 las estimaciones lo ubicaban en el $1.35 \%$ anual. (Conapo, 2008). Respecto a la esperanza de vida, mientras en 2000 la vida media de los mexicanos se ubicaba en 73.9 años, para 2008 este valor había ascendido a 76.5 .

Una de las consecuencias más relevantes de la combinación de estas tendencias, y que particularmente compete ser considerada en el marco de este trabajo, tiene que ver con las 
transformaciones producidas como consecuencia de estas dinámicas en la estructura por edad de la población. Si la participación de la población infantil (0 a 14 años) ha comenzado a decrecer ya desde el año 2000, el grupo de jóvenes (15 a 24 años) comenzará su descenso hacia 2011. Mientras la población adulta (25 a 64 años) continuará su crecimiento hasta 2040 para luego comenzar a descender, el grupo que registrará mayor crecimiento hasta mediados del siglo XXI es el de adultos mayores (más de 65 años), que se cuadruplicará entre 2008 y 2050, pasando de cerca de 6 millones en la actualidad a casi 26 millones para mitad de siglo. (Conapo,, 2008).

Estas estimaciones determinan que para 2050 los mayores de 60 años pasarán a representar al $27.72 \%$ de la población total del país, los de 65 años y más al $21.27 \%$ de la población total y los de 70 años y más al $15.33 \%$. Estos valores se ubican muy por encima del $7.79 \%$ de la población total, $5.35 \%$ de la población total y 3.45\% de la población total que dichos grupos representaban respectivamente en 2006 (Reyes Tepach, 2006).

En términos gráficos esto supondrá el tránsito de una pirámide poblacional con una base ancha y que adopta una forma triangular, como la observada en la actualidad, hacia una en la que dicha base se ha reducido drásticamente y cuya forma se asemeja más a un hongo o una choza, como la esperada para 2050. (Conapo,, 2008).

\section{Tasa de dependencia demográfica y pobreza}

De acuerdo con estimaciones de Conapo (2008), para el segmento de la población comprendida entre los 30 y los 50 años el monto de ingresos producido por actividad laboral supera al monto destinado a consumo, tanto público como privado (Déficit del Ciclo de Vida Económico). Para los grupos más jóvenes y para los adultos mayores, en cambio, esta relación se revierte, lo que los convierte en dependientes económicos. En relación a los adultos mayores, el incremento en el monto orientado a consumo está explicado en importante medida por los mayores gastos en salud en los que dichos grupos de edad deben incurrir. Asimismo, es de destacar que a partir de los 63 años se observa un cambio sustancial en el patrón de ingresos, que supone un pasaje de los ingresos salariales a los ingresos de fuentes propias (Conapo, 2008).

A partir de los datos anteriores se pueden determinar las consecuencias generales que el proceso de envejecimiento de la población implicará en términos de transferencias de ingresos entre diferentes grupos etarios. En relación a la población en su conjunto, la tasa de dependencia demográfica mide la relación entre la población en edad de trabajar (15 a 59 años) y aquella que no trabaja (menores de 15 y mayores de 60). Dicha tasa disminuirá entre tanto el segmento de la población en edad productiva continúe creciendo, mientras que lo opuesto podrá observarse en escenarios en los que se verifican aumentos sustantivos de los segmentos de población infantil y/o juvenil y/o de adultos mayores. 
Si observamos los datos para México, la tasa de dependencia demográfica registra una caída sostenida desde 1970 y, aunque de modo menos pronunciado, continuará su descenso hasta el año 2025 aproximadamente. ( Conapo, 2008). En 2006 por cada 100 personas en edad de trabajar existían 60 que no, al tiempo que para 2015 serán 53 las personas no activas por cada 100 en edad laboral. Sin embargo, los efectos del cambio de tendencia serán evidentes para 2050, cuando por cada 100 personas en edad laboral existan alrededor de 80 que no trabajen. (Reyes Tepach, 2006). El crecimiento del segmento de la población de adultos mayores será lo que explique dicho cambio: mientras que para 2006 existían 12.5 adultos mayores por cada 100 personas en edad laboral, para 2050 ese número se habrá casi quintuplicado, estimándose la existencia de 50 adultos mayores por cada 100 personas en edad laboral. Una de las consecuencias directas del aumento de la tasa de dependencia será una disminución del ingreso per cápita, como resultado de la mayor incidencia que tendrán los ingresos percibidos por los adultos mayores que tienden en general a ser menores que los ingresos promedios.

Trasladándonos al nivel de los hogares, el envejecimiento paulatino de la población supondrá un incremento de la proporción de hogares que tienen a un adulto mayor como jefe del hogar y hogares que tienen al menos un adulto mayor como uno de sus miembros. Ya para el año 2002 un cuarto de los hogares mexicanos contaba con, al menos, un adulto mayor, siendo que dicha proporción ascendía a 30.9\% en áreas rurales, en donde además 2 de cada 3 hogares son pobres de capacidades (Gomes, 2006). Asimismo, del total de personas mayores de 60 años, $61.4 \%$ era jefes de sus hogares, $24.5 \%$ cónyuges y $14.1 \%$ tienen algún grado de parentesco con el jefe. (Gomes, 2006).

No está claro que exista una relación definida entre los hogares cuyos jefes son adultos mayores y las condiciones de pobreza del hogar (Gomes, 2006). Sin embargo, sí es posible establecer que al considerar hogares pobres se observa una distribución bimodal en cuanto a las edades de los jefes de hogar, ubicándose las mayores frecuencias entre los 30 y 34 años y luego en los 70 años. En cualquier caso, no parece ser la presencia de adultos mayores en el hogar lo que determina la condición de pobreza del mismo sino el aporte en términos monetarios que el adulto mayor realiza al ingreso total del hogar (Gomes, 2006). Un análisis de los hogares con adultos mayores permite diferenciar aquellos en los que el adulto mayor realiza aportes en manera conjunta con los miembros adultos del hogar (44.10\%), en un 38.2\% es el único que aporta mientras que en $17.7 \%$ el adulto mayor es dependiente. Comparando estas cifras con los niveles de pobreza es posible observar que a mayor nivel de pobreza más frecuente es el caso en el que el/los adultos mayores del hogar no realicen ningún aporte o que el ingreso por ellos generados resulte sólo una porción reducida del ingreso total del hogar (Gomes, 2006). Entonces la pobreza de los hogares no aparece asociada a la presencia de adultos mayores sino de niños, siempre y cuando el adulto mayor continúe jugando un rol económico activo (hecho que puede generalmente implicar que éste no se jubile y continúe trabajando) en el hogar, más allá del tamaño del mismo. En cuanto a la composición del hogar se observa que los adultos mayores tienden a conformar hogares unipersonales o vivir en pareja cuando no deben enfrentar situaciones de 
pobreza, observándose una mayor propensión a que los adultos mayores sean parte de hogares extensos en el caso de hogares pobres (Gomes, 2006).

Una comparación de las pirámides de población según se trate de pobres y no pobres muestra que las mayores diferencias se observan en los grupos etarios que están en la base de la pirámide, siendo dicha base mucho más ancha para la población pobre. Sin embargo, se observa un mayor índice de dependencia entre los adultos mayores pobres respecto de los no pobres (Zuñiga y Gomes, 2002).

Un último punto remite a la certeza que dentro del tipo de ingresos que los adultos mayores reciben y utilizan para sostener su propio hogar o aportar a los ingresos familiares, el acceso a una pensión marca una diferencia sustantiva, aumentando de manera significativa las posibilidades de ser parte de hogares pobres en caso de no contar con esa fuente de ingresos (Gomes, 2006). Así, un análisis de los ingresos efectivamente percibidos por los adultos mayores pobres y no pobres demuestra que mientras los primeros deben acudir al trabajo por cuenta propia, a la obtención de subsidios y a remesas familiares para sobrevivir, los no pobres se diferencian de los anteriores a consecuencia del mayor acceso que tienen a pensiones y jubilaciones (Zuñiga y Gomes, 2002).

El progresivo envejecimiento de la población impondrá un límite temporal a la actual vigencia del bono demográfico. De no aprovecharse esta «ventana de oportunidad» para generar las condiciones que aseguren un crecimiento económico sostenido, las consecuencias serán profundamente negativas.

En relación a las dinámicas familiares, el envejecimiento de la población no necesariamente supondrá un aumento del porcentaje de familias en condiciones de pobreza, aunque en el caso de las familias más vulnerables esto implicará que los adultos deban continuar trabajando en aquellos casos en los que no puedan acceder a una pensión o jubilación ${ }^{6}$.

Retomando lo planteado al inicio, las transformaciones en la estructura etaria supondrán un incremento significativo de los sectores de la población por sobre los 60 años, mientras que al mismo tiempo se podrá observar una disminución de la población infantil y juvenil. La reducción que será experimentada por estos grupos etarios sin duda disminuirá las presiones hoy día presentes en relación al acceso a la educación, liberando recursos que pueden ser reorientados. Sin embargo, al mismo tiempo aumentará la necesidad de consolidar un sistema de salud que pueda atender mejor a la población en edad avanzada y extender el acceso de los adultos mayores a pensiones y jubilaciones.

6 Según estimaciones de Conapo con base en datos del Censo General de Población y Vivienda de 2000, los adultos mayores de 60 años tenían para dicho año casi un 30\% de participación en la PEA, ocupándose un $20 \%$ en el sector formal y un $80 \%$ en el sector informal de la economía. 


\section{CAMBIO DEMOGRÁFICO Y LIMITACIONES DEL SISTEMA VIGENTE DE PENSIONES}

Hemos observado que la posibilidad de contar con un ingreso a través del sistema de pensiones resulta central para que los adultos mayores puedan escapar a situaciones de pobreza y marginación. La reforma experimentada por dicho sistema a partir de la promulgación de una nueva ley de Seguro Social en diciembre de 1995 y la entrada en vigencia de un nuevo sistema en 1997 -obligatorio en esa primera etapa para todos los trabajadores afiliados al Instituto Mexicano de Seguro Social (IMSS)- supuso el pasaje de un esquema de reparto a uno de capitalización individual.

Dicha reforma coincidió con procesos similares observados en otros países latinoamericanos, orientados en todos los casos a darle una mayor participación al mercado e intentando revertir los profundos déficits asociados a la operación de los sistemas de pensiones.

Tal como es de extendido conocimiento, los sistemas de pensiones basados en la lógica del reparto financian las pensiones y jubilaciones pagadas a los adultos mayores que han cumplido con una cierta cantidad de años de actividad laboral con los aportes que realizan al sistema los trabajadores en situación laboral activa. De este modo, la viabilidad del sistema depende de manera significativa de la proporción entre el número de aportantes y el de quienes reciben los beneficios. Las tendencias hacia el envejecimiento poblacional, por un lado, y el incremento de la esperanza de vida, por el otro, han sido señalados como significativas amenazas para la sustentabilidad de dichos sistemas de reparto, en tanto la conjunción de dichas dinámicas supone en primer lugar la reducción de la ratio activos/pasivos al tiempo que se extiende el período durante el cual los derecho-habientes reciben su jubilación o pensión ${ }^{7}$.

En cierta medida, las reformas orientadas a elevar la edad mínima que los trabajadores deben alcanzar para poder acceder a los beneficios de una jubilación y la promoción de sistemas de capitalización individual han sido justificadas como intentos de revertir los efectos negativos derivados del envejecimiento poblacional. El sistema de capitalización individual que vino a sustituir al de reparto anteriormente vigente se sustenta en la lógica de que cada trabajador durante su etapa laboral activa debe generar su propia fuente de ahorro, recursos que recuperará en forma de jubilación o pensión al momento en el que deje de formar parte del mercado laboral. En el caso mexicano, al igual que lo que ocurre en otros países, se establece una pensión mínima garantizada que el Estado se obliga a financiar para el caso de aquellos trabajadores de menores ingresos.

7 Para el caso mexicano, la relación entre trabajadores activos y pensionados mostró un descenso de 7.1 en 1994 a 5.8 en 2006, para el caso de afiliados el IMSS, y de 7.2 en 1994 a 3.6 en 2006 para el caso de afiliados al Issste (Informe de Gobierno 2006). 
En México dichas consideraciones inspiraron la generación de una sistema de capitalización que sustituyó al sistema de reparto y que se sustentó en la obligación de los trabajadores activos -incluyendo en una primera etapa a los trabajadores afiliados al IMSS y extendiéndose en 2007 hacia los trabajadores estatales afiliados al Instituto de Seguridad y Servicios Sociales de Trabajadores del Estado (Issste) ${ }^{8}$ - de generar cuentas individuales de capitalización en las Administradoras de Fondos para el Retiro (Afores) ${ }^{9}$.

Los datos referidos al crecimiento de la cantidad de cuentas individuales desde la transformación del sistema y la evolución en la cantidad de dinero administrado por las Afores han sido generalmente presentados como una confirmación del éxito del nuevo esquema. Tal como puede observarse en la Tabla 1, la cantidad de cuentas casi se cuadruplicó entre 1997 y 2007, al tiempo que los fondos totales administrados por el sistema se ubicaron en un $13.8 \%$ del PIB.

Tabla 1.

Evolución de cantidad de cuentas y fondos acumulados

\begin{tabular}{|c|c|c|}
\hline & $\begin{array}{c}\text { Cantidad de cuentas } \\
\text { (Millones) }\end{array}$ & $\begin{array}{c}\text { Fondos acumulados Afores } \\
\text { como \% del PIB }\end{array}$ \\
\hline 1998 & 13.8 & 5 \\
\hline 1999 & 15.6 & 6.1 \\
\hline 2000 & 17.8 & 7 \\
\hline 2001 & 26.5 & 8.7 \\
\hline 2002 & 29.4 & 9.6 \\
\hline 2003 & 31.4 & 10.5 \\
\hline 2004 & 33.3 & 11 \\
\hline 2005 & 35.3 & 12.1 \\
\hline 2006 & 37.4 & 13.2 \\
\hline 2007 & 38.5 & 13.8 \\
\hline
\end{tabular}

Fuente: Informe de Gobierno 2007. Presidencia de México.

8 El del IMSS y el del Issste resultan ser los regímenes de seguridad social con mayor cantidad de derechohabientes y, por lo tanto, aquellos cuya reforma ha tenido un impacto más profundo. Todavía continúan existiendo, sin embargo, otros regímenes con reglas y características particulares que no han sido aún objeto de reformas sustantivas. Entre ellos es posible mencionar los regímenes de trabajadores del sector paraestatal, de las universidades, de los gobiernos estatales y del sector bancario.

9 El nuevo sistema se propuso asimismo como un remedio para las limitaciones en cuanto a la portabilidad de los aportes existentes en el sistema de reparto anteriormente vigente. 
Sin embargo, dichas cifras esconden cuestiones que deben ser analizadas para entender los potenciales impactos futuros del sistema de capitalización. En primer lugar, del total de cuentas, sólo un $40 \%$ se encuentran activas, es decir que reciben aportes regularmente. El resto aparecen como inactivas o sólo reciben aportes de manera irregular. Por otro lado, del total de la población activa, el 65.8\% se encontraba por fuera del sistema de seguridad social, mientras que ese valor se ubicaba en $43.6 \%$ para el caso de los asalariados. Estas cifras demuestran la importancia que la economía informal continúa teniendo en la generación de empleos, tendencia que no ha podido ser revertida en los últimos años. Según datos del Banco de México (Alcaraz et al 2008), para fines de 2004 casi un 45\% del total de la población ocupada en el ámbito urbano cumplía sus tareas en el sector informal.

En el caso de los adultos mayores, sólo algo más del 30\% recibe una pensión del IMSS o Issste (aproximadamente 3 millones de pensiones sobre un total de casi 9 millones de individuos de 60 años o más) ${ }^{10}$, valor que demuestra la extremadamente acotada cobertura alcanzada por el sistema.

Dado el grado de amenaza que el envejecimiento poblacional supone para la sustentabilidad del sistema de reparto, la principal razón para el reemplazo de este por un sistema de capitalización individual parece estar en el alivio que a futuro dicha reforma supondrá para las cuentas públicas. Si bien en el corto plazo el gobierno federal continuará financiando los costos generados como consecuencia del «período de transición»-lo que supone que el IMSS e Issste continúen proveyendo los fondos para pagar las pensiones de quienes se retiraron con anterioridad al cambio del sistema- en el largo plazo la presión fiscal que actualmente supone el sostenimiento del sistema tenderá a descender.

Sin embargo, los efectos no parecen ser positivos cuando otros aspectos son considerados, en especial en lo que refiere a la limitada cobertura del sistema. La reforma no parece haber apuntado a generar las condiciones para revertir esta realidad. En primer lugar, la imposibilidad demostrada por la economía mexicana de absorber en el mercado de trabajo formal a las crecientes generaciones que en el presente se incorporan a la vida activa determinará que dichos individuos no puedan contar con una pensión al momento de pasar a formar parte del grupo de adultos mayores. En segundo lugar, las experiencias internacionales han demostrado que la evasión suele ser más elevada en los sistemas de capitalización y la mayor transparencia generada a partir de la posibilidad del trabajador de controlar periódicamente su fondo de aportes se ve contrapesada por la posición de debilidad en la que el trabajador se encuentra en el caso que su empleador no realice los aportes correspondientes. En tercer lugar, la discontinuidad en los aportes como consecuencia de los vaivenes del mercado laboral y la evasión implicará que muchos trabajadores deban extender su vida laboral para poder alcanzar la cantidad de años de

10 Datos de Conapo e Informe de Gobierno 2006. 
aportes necesarios para poder acceder a la renta vitalicia. En cuarto lugar, los sistemas de capitalización tienden a aumentar las desigualdades entre los beneficiarios: así como en la actualidad 2 de cada 3 pensiones resultan insuficientes, es esperable que dicha tendencia se consolide o incluso empeore a futuro.

Las perspectivas resultan preocupantes si vuelve a destacarse el impacto que el acceso a las pensiones tiene en términos de la reducción de las posibilidades de caer en situaciones de pobreza. Una acción de gobierno orientada a garantizar esquemas de ingresos mínimos para los adultos mayores, atendiendo especialmente a aquellos que se encuentren por fuera del sistema de seguridad social, resulta ya perentoria y será aún más necesaria en las décadas por venir.

\section{EL ENVEJECIMIENTO POBLACIONAL Y LA PRESTACIÓN DE SERVICIOS DE SALUD}

Tal como diferentes análisis han señalado, otra de las importantes consecuencias derivadas del envejecimiento poblacional está dada por las crecientes presiones que el incremento de la esperanza de vida genera en relación a la provisión de servicios de salud. El crecimiento del grupo de edad de adultos mayores supone, por un lado, un incremento de la población con mayores necesidades de atención médica y, por el otro, un seguro incremento en las tasas de población con enfermedades crónicas y degenerativas e incapacidad.

En la actualidad diferentes sistemas cubren las necesidades de salud de la población de adultos mayores según su condición de derechohabiencia, aunque existe una porción significativa de dicho grupo etario que no cuenta con protección de ningún tipo. De acuerdo con los datos que surgen de la Encuesta Nacional de Salud y Nutrición 2006, casi un 40\% de la población mayor de 60 años es derechohabiente del IMSS, aproximadamente un 9\% del Seguro Popular, un 8\% del Issste y un $4 \%$ de otras instituciones o prestadores privados, mientras que un $40 \%$ aproximadamente no es derechohabiente de ninguna institución. (Shamah Levy et al 2008).

Además de limitada, la cobertura presenta importantes problemas de calidad. Tanto el IMSS como el Issste tienen sostenidos déficits operativos en sus áreas de cobertura médica, lo cual impacta en la provisión del servicio como consecuencia de la falta de insumos en las clínicas y hospitales, así como en obras de modernización y adecuación de las instalaciones y equipos existentes.

La cobertura brindada por las mencionadas instituciones en zonas rurales resulta ser extremadamente limitada, alcanzando a solo 3\% del total de la población ocupada. Por otro lado, la creciente demanda genera la saturación de las capacidades de prestación. Esto ha llevado, por un lado, al aumento de las quejas de los derechohabientes frente a organismos como la Comisión Nacional de Derechos Humanos $(\mathrm{CNDH})$ y, por el otro, a una tendencia cada vez mayor de 
los derechohabientes de ambas instituciones a acudir a la medicina privada. De acuerdo con datos del Instituto Nacional de Estadística, Geografía e Informática (Inegi), para 2004 la mitad de los derechohabientes del IMSS prefería acudir a médicos privados antes que utilizar los servicios del Instituto, mientras que lo mismo ocurría con 3 de cada 5 derechohabientes del Issste. (García Ponce, 2006).

Si bien el Seguro Popular impulsado por el gobierno federal desde 2002 ha servido para cubrir las necesidades de un porcentaje paulatinamente creciente de la población (pasando de poco menos de 300 mil asegurados en 2002 a un total de casi 5.200.000 en 2006), su incidencia total sigue siendo limitada.

Finalmente, la población que no se encuentra cubierta por ningún seguro de salud ha sido objeto de diferentes políticas focalizadas, tanto de programas orientados específicamente a proveer servicios de salud a poblaciones particulares (como el caso de «Comunidades Saludables» o IMSS-Oportunidades), como de programas sociales que incluyen algún componente de salud entre sus objetivos (principalmente el programa Oportunidades, bajo la órbita de la Sedesol).

Las condiciones actuales no parecen proveer razones optimistas para pensar que en las próximas décadas las crecientes demandas que se generarán como consecuencia del envejecimiento poblacional podrán ser adecuadamente atendidas. Especial atención requerirá la porción de la población que no se encuentra protegida por un seguro de salud y que en general coincide con condiciones de existencia más desfavorables. Proveer las condiciones para mejorar la atención de instituciones como el IMSS y el Issste aparece asimismo como imprescindible para dar cuenta del desafío que viene.

\section{PROGRAMAS DE EDUCACIÓN Y ASISTENCIA A LA NIÑEZ}

Si bien el envejecimiento poblacional generará mayores demandas en relación con el segmento de adultos mayores, al mismo tiempo relajará las presiones derivadas de la prestación de servicios a la población infantil al disminuir el peso de este grupo etario.

Este contexto más favorable deberá ser aprovechado para promover acciones que den cuenta de las condiciones negativas que todavía afectan a la población infantil. De acuerdo con el Conteo de Población de 2005 algo más de la mitad de la población infantil carecía de seguro de salud (Conapo, 2007). El Seguro Médico para una Nueva Generación creado en 2006 en el marco del Seguro Popular se planteó como una acción en ese sentido, aun cuando sólo pueden incorporarse al mismo familias sin seguro social con niños nacidos luego del $1^{\circ}$ de enero de 2006 (la meta del programa es lograr asegurar al 100\% de la población con esas características para el final de la administración del actual Presidente Felipe Calderón que soliciten ser incluidas en el programa). 
Una porción importante de la población infantil se encuentra inmersa en condiciones de marginación y pobreza. Cerca de 6.4 millones de niños viven en municipios de alta o muy alta marginación. Al mismo tiempo, la Encuesta Nacional sobre el Uso del Tiempo 2002 reveló que de cada diez niños de entre 12 y 14 años uno realiza trabajo para el mercado y tres trabajos domésticos. Dicha realidad pretende ser atacada en la actualidad a partir de la acción de diversos programas sociales coordinados por la Secretaría de Desarrollo Social (Sedesol) y a través de diversas acciones asistenciales bajo la órbita del Sistema Nacional de Desarrollo Integral de la Familia (Sndif). En los próximos años será necesario estudiar la posibilidad de desarrollar iniciativas orientadas, por ejemplo, a la implementación de sistemas de ingreso mínimo garantizado por niño con el objetivo de generar condiciones más favorables para el desarrollo integral de la población infantil.

Por último, es de destacar que en lo referido a la educación, la cobertura ha alcanzado niveles casi universales para la educación preescolar, primaria y secundaria. (91,97 y 90\% respectivamente). Sin embargo, aún es necesario promover acciones y programas orientados a mejorar la calidad de la enseñanza y preparar mejor a los jóvenes para las siguientes etapas de sus trayectorias educativas. Los magros resultados alcanzados por los estudiantes en las evaluaciones internacionales durante los últimos años refuerzan la necesidad de avanzar en este sentido.

\section{CONCLUSIONES}

Hemos buscado analizar de qué manera el proceso de envejecimiento experimentado por la sociedad mexicana durante las últimas décadas plantea nuevos desafíos para las políticas públicas orientadas a atender a grupos etarios particulares de la población.

En lo referente a los programas de gobierno enfocados en los adultos mayores, el limitado alcance del sistema de seguridad social establece un déficit sustantivo del que el Estado debe dar cuenta. Tal como hemos destacado, sólo una porción menor de los mayores de 65 años cuentan con algún tipo de ingreso por jubilaciones o pensiones, y casi la mitad de los mismos no posee ningún tipo de cobertura médica. Esta situación parece ser la consecuencia inevitable del peso sustantivo que la economía informal continúa teniendo y de la limitada generación de puestos de trabajo observada durante los últimos años en el sector formal. De este modo una porción mayoritaria de la población debe transitar durante la mayor parte (o la totalidad) de su vida laboral por empleos precarios dentro de dicho sector, viéndose privados de la protección otorgada por la legislación laboral y no pudiendo por lo tanto constituirse en derechohabientes de la seguridad social. Como consecuencia de lo anterior, al finalizar sus años activos dichos individuos no pueden acceder ni al derecho de un ingreso garantizado, vía pensión, que ayude a costear su supervivencia, ni a la protección sanitaria provista por las instituciones de la seguridad social. Así, la situación de vulnerabilidad en la que de por sí se encuentran quienes han llegado a esta etapa de la vida se ve agravada por el no poder hacer ejercicio de estos derechos fundamentales que el Estado debería garantizar. 
Las iniciativas gubernamentales puestas en práctica durante los últimos años no parecen haber logrado revertir completamente esta realidad. Por un lado, la reforma del sistema provisional estuvo más orientada a atacar el déficit presupuestario del sistema vigente más que a generar condiciones para la expansión de su cobertura. Asimismo, los esquemas de transferencia focalizados a este grupo etario (tales como el componente «Adultos mayores» de Oportunidades o el Programa Adultos mayores de 70 años y más, creado hace algunos años por la Sedesol) por el momento no han logrado más que un alcance limitado y proveen una cantidad de recursos muy por debajo de los necesarios para asegurar condiciones de vida dignas.

Por otro lado, el déficit en relación con la atención de la salud de los adultos mayores sólo se ha visto revertido en parte como resultado de la puesta en marcha de programas como el Seguro Popular de Salud o la extensión del Programa Oportunidades. El carácter focalizado del segundo determina que sólo una porción de quienes no son derechohabientes sean beneficiarios, al tiempo que las prestaciones médicas se orientan fundamentalmente a acciones de carácter preventivo. En cuanto al Seguro Popular, sus alcances también son aún limitados. El hecho de que se trate de un programa de afiliación voluntaria y que no cuente con un componente específicamente orientado al tipo de cuadros que afectan al sector de adultos mayores (a diferencia de lo que ocurre en relación con los recién nacidos y sus madres) deja en evidencia el no cumplimiento del derecho a la salud para este grupo etario.

Teniendo en cuenta que el segmento de adultos mayores será el que a mayor ritmo crecerá durante los últimos años, de no diseñarse políticas de carácter más integral, que logren revertir la situación de privación experimentada por una significativa cantidad de miembros de dicho grupo, la situación será todavía más crítica en los años por venir. La gravedad de dicha situación parece profundizarse por el impacto negativo que las reformas y programas recién mencionados han tenido en relación al contrato intergeneracional que coadyuva a generar cohesión social. La reforma previsional ha anulado el supuestos básico sobre el que se erigieron los sistemas de seguridad social, la idea solidaria de que las generaciones laboralmente activas son las que, a partir de sus aportes previsionales, solventan la supervivencia de las generaciones de adultos mayores ya retirados bajo el entendimiento de que las nuevas generaciones harán lo propio en el futuro. Con el nuevo esquema dicha solidaridad intergeneracional se ve truncada, en tanto se traslada a cada individuo la responsabilidad de generar los recursos para sustentar su propio retiro. Como en otros aspectos de las reformas estructurales llevadas a cabo en el país y en la región, todo el peso recae en las personas a quienes se las convierte en responsables (culpables) de no lograr un trabajo digno (ahora decente) y de no poder, en consecuencia, realizar aportes jubilatorios que permitan un retiro decoroso.

En contraposición, la reducción del peso de las generaciones más jóvenes en las próximas décadas genera la oportunidad para proponer una transformación cualitativa a los programas orientados a dicho grupo etario. En lo que específicamente refiere a la política educativa, si las últimas décadas estuvieron signadas por los esfuerzos para que el sistema lograse incorporar a la mayor 
cantidad posible de niños que alcanzaban la edad escolar, dichas presiones tenderán a hacerse menores en los próximos años. Esta situación generará las condiciones para avanzar en el mejoramiento de la calidad de la enseñanza impartida en las escuelas, modernizando los aprendizajes y vinculando a los niños de manera más profunda con las nuevas tecnologías.

\section{BIBLIOGRAFÍA}

- Alcaraz, C; D. Chiquiar; M. Ramos-Francia (2008). «Diferenciales Salariales Intersectoriales y el Cambio en la Composición del Empleo Urbano de la Economía Mexicana en 2001-2004» Banco de México. Documentos de Investigación No 2008-06.

- Aguilar Villanueva, L (ed.) (1995). Problemas Públicos y Agenda de Gobierno. Ciudad de México: Miguel Angel Porrua Grupo Editorial.

- CEPAL (2008). Panorama Social de América Latina 2007. Santiago de Chile: Cepal.

- Consejo Nacional de Población (2008). «Informe de México: El cambio demográfico, el envejecimiento y la migración internacional en México», informe presentado al Comité Especial sobre Población y Desarrollo en el XXXII Período de Sesiones de la Comisión Económica para América Latina y el Caribe (CEPAL), Santo Domingo, Rep Dominicana, 9 al 13 de Junio.

- Diario Oficial de la Federación (2008). «Ley de los Derechos de las Personas Adultas Mayores». Cámara de Diputados del H. Congreso de la Unión. México.

- Diario Oficial de la Federación (2004). «Ley General de Desarrollo Social». Cámara de Diputados del H. Congreso de la Unión. México.

- Diario Oficial de la Federación (2000). «Ley para la Protección de los Derechos de Niños, Niñas y Adolescentes». Cámara de Diputados del H. Congreso de la Unión. México.

- Diario Oficial de la Federación (1993). «La Ley General de Educación». Cámara de Diputados del H. Congreso de la Unión. México.

- García Ponce, A. (2006). «Panorama de la Seguridad Social en México y los Retos sindicales» Comisión de Seguridad Social. Cámara de Diputados LX Legislatura.

- Gobierno Nacional de los Estados Unidos Mexicanos, Presidencia de la República (2007). Informe de Gobierno 2007.

- Gobierno Nacional de los Estados Unidos Mexicanos, Presidencia de la República (2006). Informe de Gobierno 2006.

- Gomes, C. (2006). «Pobreza, familia y envejecimiento poblacional en México» en Carlos de la Torre Martínez (coord) Derecho a lo no discriminación Instituto de Investigaciones Jurídicas, UNAM. México. 
- Kauffer Michel E.F (2002). «Las políticas públicas: algunos apuntes generales» Ecofronteras. 16: 2-5. Ciudad de México, México.

- Lowi, T (1972). «Four Systems of Policy, Politics and Choice» in Public Administration Review, Vol. 32, No. 4 (Jul. - Aug) Blackwell Publishing.

- Lowi, T (1964). «American Business, Public Policy, Case-Studies and Political Theory» in World Politics, Vol. 16, No. 4 (Jul y) Baltimore: The Johns Hopkins University Press.

- Oficina del Alto Comisionado de las Naciones Unidas para los Derechos Humanos en México (2003). Diagnóstico sobre la situación de los Derechos Humanos en México. Ciudad de México: Mundi-Prensa México.

- Oficina del Alto Comisionado de las Naciones Unidas para los Derechos Humanos (2006). Preguntas Frecuentes sobre el Enfoque de Derechos Humanos en la Cooperación para el Desarrollo. Ginebra, Suiza.

- Oszlak, O. y Guillermo O'Donnell, (1976). Estado y Políticas Estatales en América Latina: Hacia una Estrategia de Investigación, Doc. CEDES/G. E. CLACSO № 4.

- Palumbo, D.J. (ed) (1987). The Politics of Program Evaluation. Sage, Newbury Park, California. Estados Unidos.

- Parsons, W (2007). Políticas públicas: una introducción a la teoría y la práctica del análisis de políticas públicas. FLACSO-México y Miño y Dávila Editores. Ciudad de México, México y Buenos Aires, Argentina.

- Reyes Tépach, M. (2006). «Análisis demográfico y socioeconómico de la población de ADULTOS MAYORES de México, 2006-2050» Servicios de Investigación y Análisis de la Cámara de Diputados. Subdirección de Economía.

- Shama-Levy, T; L Cuevas-Nasu; V. Mundo-Rosas; C. Morales-Ruán; L. Cervantes-

- Turrubiates; S. Villalpando-Hernández (2008). «Estado de salud y nutrición de los adultos mayores en México: resultados de una encuesta probabilística nacional» en Salud Pública de México. Vol 50. No 5. Septiembre-octubre.

- Zuñiga. E y C. Gomes (2002). «Pobreza, curso de vida y envejecimiento poblacional en México» en Conapo La situación demográfica en México. 